\title{
Management of Residual Left Atrial Appendage Stump with a Second Epicardial Clip.
}

\author{
Saqib Masroor ${ }^{1}$ and Abdullah Nasif ${ }^{1}$ \\ ${ }^{1}$ The University of Toledo Department of Surgery
}

December 4, 2021

\begin{abstract}
Epicardial exclusion of LAA has gained increasing popularity since its introduction in 2009. While the clip has demonstrated a success rate ranging from $93.9-100 \%$ in excluding the LAA, one significant complication is incomplete exclusion due to residual stump $(>1 \mathrm{~cm})$ as a result of the clip being deployed too distally. We describe here the first reported case of implanting a second clip underneath the first clip to obliterate the residual stump for a 71-year-old male with AF evaluated for intervention for stroke risk reduction.
\end{abstract}

\section{Introduction:}

Epicardial exclusion of LAA has gained increasing popularity since its introduction in 2009. However, there are single center studies of this technique for standalone stroke risk reduction in patients with $\mathrm{AF} .{ }^{1-3}$ While the clip has demonstrated a success rate ranging from 93.9-100\% in excluding the LAA, one significant complication is incomplete exclusion due to residual stump greater than $1 \mathrm{~cm}$ as a result of clip being deployed too distally. ${ }^{1}$ We describe here a solution to that complication, which is the first reported case of implanting a second clip underneath the first clip to obliterate the residual stump.

\section{Methods and methods:}

Patient chart was reviewed for complete history and details of his operative details and postoperative course. The Institutional Review Board (IRB) approval was not obtained, since single case report with deidentified data does not require IRB approval. The patient provided informed written consent for the publication of the study data.

\section{Results:}

A 71-year-old male with morbid obesity $(\mathrm{BMI}=39)$, hypertension, AF, history of ischemic stroke, diastolic heart failure and recent life-threatening hemorrhage after angiography, was evaluated for intervention for stroke risk reduction. He had a $\mathrm{CHA}_{2} \mathrm{DS}_{2}$-VASc score of 5 and HAS-BLED score of 3. He underwent thoracoscopic exclusion of LAA with the Atriclip PRO 2 device (Atricure Inc., Cincinnati, OH) (Figure 1). The technique involved single lung ventilation of the left side, a $5 \mathrm{~mm}$ camera port in the $5^{\text {th }}$ and a $12 \mathrm{~mm}$ port in the $7^{\text {th }}$ intercostal space in the mid-axillary line and a $5 \mathrm{~mm}$ working port in $7^{\text {th }}$ intercostal space anterior to the anterior axillary line. $\mathrm{CO}_{2}$ insufflation was used and pericardium was opened posterior to the phrenic nerve using Harmonic Scalpel (Ethicon. Raritan, NJ). The LAA was exposed with endoscopic kittners, taking care not to grasp the appendage at this stage to reduce the risk of bleeding. A $45 \mathrm{~mm}$ Atriclip Pro 2 device (with a closed ring) was threaded over the tip of the LAA and carefully pulled down to the base. Once TEE showed no residual stump, the device was deployed. However, after removal of the delivery system, a significant residual stump was seen (Figure 1). It was decided to obliterate this residual stump with an open-mouth Atriclip Pro V device (Figure 2). Holding the tip of the LAA by an endoscopic grasper, 
the second clip was introduced underneath the first clip and by gentle rocking motion, it was advanced medially to traverse the base of LAA (Video 1). TEE confirmed successful obliteration of residual stump. Patient was extubated in the operating room and was discharged home on the $3^{\text {rd }}$ postoperative day.

\section{Discussion:}

Left atrial appendectomy to prevent embolism in patients with rheumatic heart disease and atrial fibrillation was first reported by Madden in $1949 .{ }^{4}$ In order to prevent embolism in patients with AF undergoing LAA excision/exclusion, the surgical technique is important. Years later, in his report of 306 patients who underwent maze procedure and followed for up to 11.5 years, Dr. Cox reported two early strokes with an incidence of $0.7 \%$ and only one more stroke in the follow-up period. ${ }^{5}$ This benefit was ascribed to the maze procedure restoring sinus rhythm and atrial transport, and secondarily to obliteration of the LAA.

One of the complications of the clip is "too distal deployment" on the LAA, leaving a residual stump greater than $1 \mathrm{~cm}$. While the $1 \mathrm{~cm}$ figure is an arbitrary number and its impact on future risk of stroke is undetermined, it remains a considerable obstacle towards a wide acceptance of this technique in the physician community. To this end, it is important that implanting surgeon do all they can to make sure the clip is deployed at the base of the LAA. The LAA may appear obliterated on the TEE due to external pressure from the delivery system during deployment of the clip. Once the clip is deployed and the delivery system disconnected the stump may bounce back outward to show its real size. It is also important that the appendage be evaluated in multiple different views to make sure of obliteration. If recognized in the operating room, such as happened in our case, one can deploy an open-mouth V-Shaped clip under the previous clip. This is the first such report of successful use of a second clip to obliterate the residual stump. Because the Atriclip Pro V is more expensive, we only keep it as a backup for such situations (because of its higher price) but continue to use the more economical Atriclip Pro 2 for routine thoracoscopic clipping. In conclusion, it is important to obliterate the LAA as close to the base as possible. If there is a residual stump of a significant size, approaching $1 \mathrm{~cm}$, a second clip can be deployed under the first clip to obliterate the LAA completely.

\section{Conclusion:}

When excluding LAA with an epicardial clip, care must be taken to leave no residual stump behind before deploying the clip. In case of a residual stump, a second clip can be applied in select cases, depending on the anatomy of the LAA.

\section{Figure/Video legend:}

Figure 1. Long chicken wing type LAA (diamond), with $45 \mathrm{~mm}$ Atriclip Pro 2 (star). TEE shows residual stump of LAA.

Figure 2. Completed exclusion of LAA with two clips: Atriclip Pro V (Triangle) and Atriclip Pro 2 (Diamond). TEE shows an obliterated residual stump.

Video 1. Thoracoscopic application of a second Atriclip proximal to the first clip on the base of LAA.

\section{References:}

[1] Ellis CR, Aznaurov SG, Patel NJ, Williams JR, Sandler KL, Hoff SJ et al . Angiographic Efficacy of the Atriclip Left Atrial Appendage Exclusion Device Placed by Minimally Invasive Thoracoscopic Approach. JACC Clin Electrophysiol. 2017 Dec 11;3(12):1356-1365. Epub 2017 May 9. PMID: 29759664.

[2] Branzoli S, Marini M, Guarracini F, Pederzolli C, Pomarolli C, D'Onghia G et al . Epicardial standalone left atrial appendage clipping for prevention of ischemic stroke in patients with atrial fibrillation contraindicated for oral anticaogulation. J Cardiovasc Electrophysiol. 2020 Aug;31(8):2187-2191. Epub 2020 Jun 17. PMID: 32495408.

[3] Smith NE, Joseph J, Morgan J, Masroor S. Initial Experience with Minimally Invasive Surgical Exclusion of the Left Atrial Appendage with an Epicardial Clip. Innovations (Phila). 2017 Jan/Feb;12(1):28-32. 
PMID: 28129318.

[4] Madden JL. Resection of the left auricular appendix; a prophylaxis for recurrent arterial emboli. J Am Med Assoc. 1949 Jul 2;140(9):769-72. PMID: 18152330.

[5] Cox JL, Ad N, Palazzo T. Impact of the maze procedure on the stroke rate in patients with atrial fibrillation. J Thorac Cardiovasc Surg. 1999 Nov;118(5):833-40. PMID: 10534688.
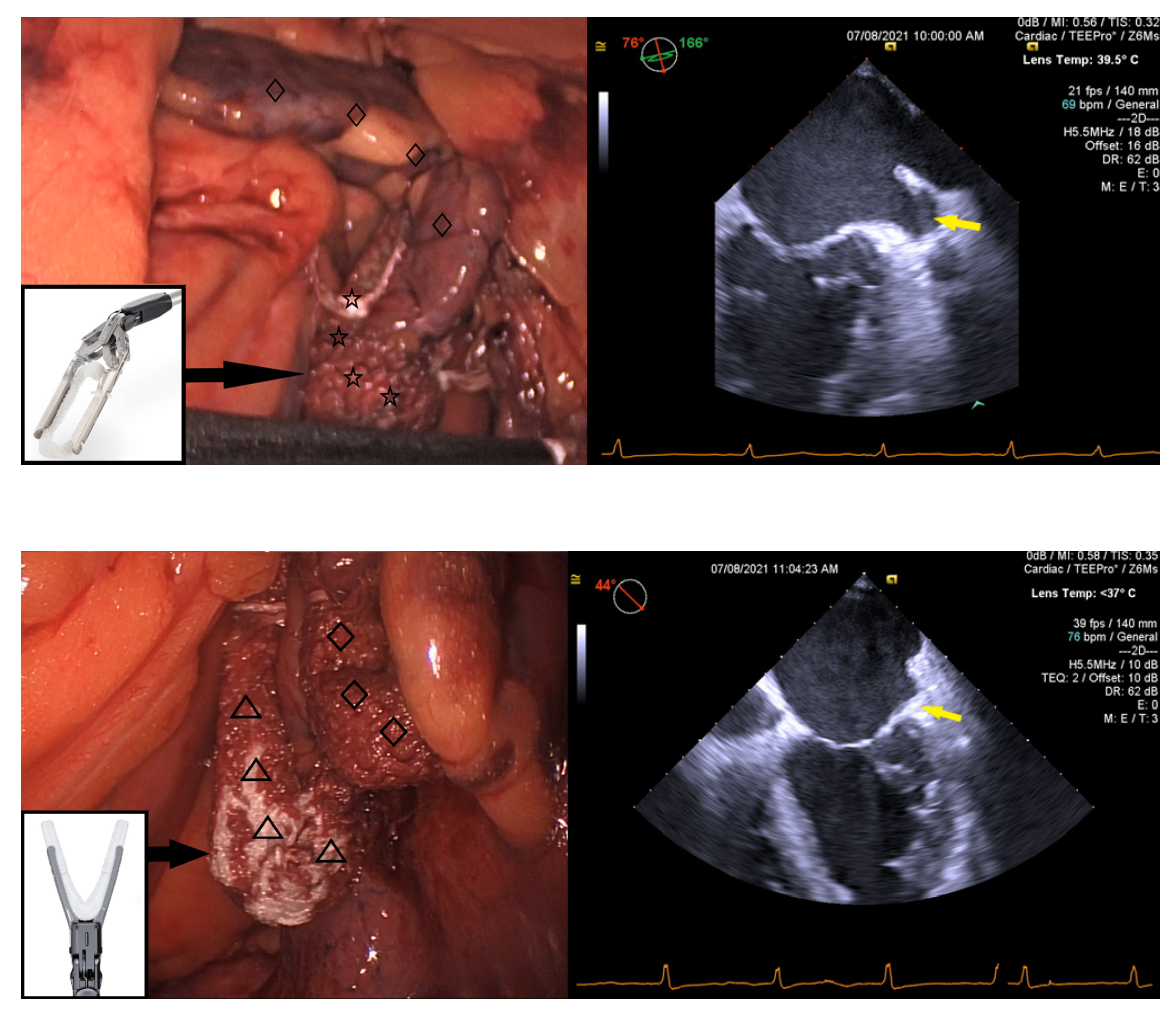

\section{Hosted file}

2nd Atrial Clip - Video 1.mp4 available at https://authorea.com/users/316008/articles/548053management-of-residual-left-atrial-appendage-stump-with-a-second-epicardial-clip 